\title{
Índice de Placa Bacteriana, Índice Gingival y Prueba de Silometría en Pacientes con Síndrome de Sjögren Primario y Secundario
}

\author{
Bacterial Plate Index, Gingival Index, and Silometry Testing \\ in Patients With Primary and Secondary Sjögren's Syndrome
}

\begin{abstract}
Silvia Arely Triana-Reyes ${ }^{1}$; Gloria Martínez-Sandoval'; Norma Idalia Rodríguez-Franco'; María Gabriela ChapaArizpe $^{1}$; Jesús Israel Rodríguez-Pulido'; Gustavo Israel Martínez-González \& Janett Riega-Torres²
\end{abstract}

TRIANA-REYES, S. A.; MARTÍNEZ-SANDOVAL, G.; RODRÍGUEZ-FRANCO, N. I.; CHAPA-ARIZPE, M. G.; RODRÍGUEZPULIDO, J. I.; MARTÍNEZ-GONZÁLEZ, G. I. \& RIEGA-TORRES, J. Índice de placa bacteriana, índice gingival y prueba de silometría en pacientes con síndrome de sjögren primario y secundario. Int. J. Odontostomat.,15(2):449-453, 2021.

RESUMEN: El síndrome de Sjögren (SS) es una enfermedad crónica autoinmune presente en el $0.1-3.0 \%$ de la población, en la que se encuentran involucradas las glándulas salivales, trayendo consigo manifestaciones orales como caries dental y enfermedad periodontal. El objetivo de este trabajo fue evaluar el índice de placa, el índice gingival y la prueba de sialometría en pacientes con síndrome de Sjögren primario y secundario. Fueron evaluados clínicamente 40 pacientes con SS primario $(n=20)$ y SS secundario $(n=20)$, ambos grupos diagnosticados con periodontitis crónica. Se registró el índice de placa de Quigley-Hein modificado por Turesky, el índice gingival de Löe y la prueba de silometría estimulada, recolectando saliva parotídea con el dispositivo Carlson-Crittenden. Los pacientes con SS primario tuvieron un índice de placa de $(3,53 \pm 0,5954)$ e índice gingival $(2,41 \pm 0,2608)$. En pacientes con SS secundario el índice de placa fue $(1,62 \pm 0,3795)$ y el índice gingival $(1,48 \pm 0,3994)$, con diferencia significativa $(p=0,0001)$ entre ambos grupos. El flujo salival se elevó en pacientes con SS secundario. El presente estudio concluye que el índice de placa y el índice gingival fueron mayores en pacientes con SS primario comparados con pacientes con SS secundario. La prueba de sialometría fue mayor en pacientes con SS secundario.

PALABRAS CLAVE: Síndrome de Sjögren, índice de placa, índice gingival, sialometría estimulada, periodontitis.

\section{INTRODUCCIÓN}

La hiposalivación es un padecimiento frecuente que afecta las condiciones de la cavidad oral al disminuir el flujo salival (Yoshimoto et al., 2013), la cual se presenta en el $10 \%$ de la población con mayor prevalencia en adultos mayores (Rodríguez et al., 2017).

Al haber un decremento del flujo salival, se presenta hiposalivación con valores menores a $0,1-0,2$ $\mathrm{mL} / \mathrm{min}$ de saliva en reposo o por debajo de $0,4-0,7$ $\mathrm{mL} / \mathrm{min}$ de saliva total estimulada (Donat et al., 2004), a diferencia de xerostomía, la cual es la sensación subjetiva de boca seca, sin manifestarse disminución del flujo salival (Jensen et al., 2010).
Una de las causas de la hiposalivación es el síndrome de Sjögren (SS), una enfermedad autoinmune crónica, de etiología desconocida y de curso lento y progresivo (Rodríguez et al., 2015a), donde se estima una prevalencia de 300 a 600 por cada 100000 personas (Goldblatt \& O'Neill, 2013).

EI SS puede presentarse de forma aislada como primario (Rodríguez et al., 2015b), o puede manifestarse como secundario al expresarse con otras enfermedades autoinmunes, tales como artritis reumatoide (Berkey \& Scannapieco, 2013), lupus eritematoso sistémico, esclerosis, entre otras (Shahane \& Patel, 2014).

\footnotetext{
${ }^{1}$ Facultad de Odontología, Posgrado de Periodoncia, Universidad Autónoma de Nuevo León, Monterrey, Nuevo León, México.

${ }^{2}$ Hospital Universitario. Centro de Especialistas en Artritis y Reumatismo (CEAR).
} 
TRIANA-REYES, S. A.; MARTíNEZ-SANDOVAL, G.; RODRÍGUEZ-FRANCO, N. I.; CHAPA-ARIZPE, M. G.; RODRÍGUEZ-PULIDO, J. I.; MARTÍNEZ-GONZÁLEZ, G. I. \& RIEGATORRES, J. Índice de placa bacteriana, índice gingival y prueba de silometría en pacientes con síndrome de sjögren primario y secundario. Int. J. Odontostomat.,15(2):449-453, 2021.

Debido a la exocrinopatía que se presenta en el SS, las condiciones ambientales de la cavidad oral se ven favorecidas para la colonización de patógenos oportunistas (González et al., 2014), existiendo un aumento de caries dental, queilitis angular (RodríguezPulido et al., 2017), neoplasias, mucositis, candidiasis, entre otras condiciones (Deepak \& Gopakumar, 2011).

Hoy en día existe controversia acerca de la afección periodontal en pacientes con SS, donde algunos estudios han mostrado mayores niveles de placa y por lo tanto un mayor número de piezas perdidas y pérdida de inserción (Antoniazzi et al., 2009), sin embargo otros han reportado menores niveles de índice gingival e índice de placa en pacientes con xerostomía (Pers et al., 2005).

El objetivo del presente estudio fue evaluar el índice gingival, el índice de placa y la prueba de sialometría estimulada en pacientes con síndrome de Sjögren primario y secundario con periodontitis crónica.

\section{MATERIAL Y MÉTODO}

Población y diseño de estudio. En el presente estudio participaron 40 pacientes, los cual fueron divididos en dos grupos: SS primario $(n=20)$ y SS secundario $(n=20)$.

El diseño del estudio fue comparativo, abierto, observacional y transversal. Los pacientes con SS primario y secundario fueron diagnosticados por el Centro de Especialistas en Artritis y Reumatismo, Hospital Universitario de la Universidad Autónoma de Nuevo León (UANL) y referidos a participar en el estudio al Posgrado de Periodoncia e Implantología Oral, Facultad de Odontología, UANL.

Fueron incluidos pacientes con SS primario y secundario, de ambos géneros, y con un rango de edad de 40 a 80 años. Fueron excluidos aquellos pacientes que pudieran tener otro factor que involucrara hiposalivación, como tratamiento de radiación en cabeza y cuello, antecedentes del virus de la hepatitis $C$, virus de inmunodeficiencia humana, linfoma pre-existente, sarcoidosis, pacientes con alguna enfermedad de injerto contra huésped, diabetes mellitus, pacientes en tratamiento drogas anticolinérgicas y parasimpaticomiméticas o su ingesta en un tiempo menor a 4 meses.
Evaluación clínica del paciente. El examen periodontal fue realizado por un solo examinador. Los pacientes fueron evaluados para detectar bolsa periodontal y pérdida de inserción, diagnosticados con o sin periodontitis crónica, Se registró el índice de placa bacteriana y el índice gingival. Se utilizó la sonda North Carolina $15 \mathrm{~mm}$.

Índice gingival. De acuerdo al índice gingival de Löe (Löe, 1967) se evaluaron las 4 zonas de la encía marginal (vestibular, lingual, mesial y distal) en relación con el diente de todas las piezas dentarias, secando la superficie dental previamente y dando una puntuación de acuerdo a las características clínicas observadas: 0 . Encía normal.

1. Ligera inflamación y cambio de coloración, sin sangrado al sondeo, 2. Inflamación moderada, enrojecimiento y sangrado al sondeo, 3. Inflamación marcada, edema, ulceración, tendencia a hemorragia espontánea. El puntaje que se obtuvo en cada diente se sumó y se dividió entre el total de dientes examinados para obtener el valor del índice gingival de cada paciente. Una puntuación de 0,1-1,0 = inflamación leve; $1,1-2,0=$ inflamación moderada y $2,1-3,0=$ inflamación severa.

Índice de placa. Para evaluar la placa dentobacteriana se tomó como referencia el índice de placa QuigleyHein modificado por Turesky et al. (1970), la medición se enfocó sobre el tercio gingival de la superficie dental bucal y lingual de todas las piezas, excepto los terceros molares, dando un puntaje según el caso: 0 . Ausencia de placa, 1. Zonas independientes de placa en el margen cervical del diente, 2. Banda delgada continua de placa en el margen cervical, 3 . Banda de placa mayor a $1 \mathrm{~mm}$ de ancho, que cubre menos de una tercera parte de la corona, 4. La placa cubre por lo menos un tercio pero no más de dos terceras partes de la corona, 5 . La placa cubre dos tercios o más de la corona. El promedio del índice se obtuvo al dividir la suma de la puntuación total de superficies entre el número de superficies examinadas. Una puntuación de 0 a 1 = bajas cantidades de placa bacteriana y 20 más $=$ altas puntuaciones de placa bacteriana.

Prueba de sialometría estimulada. Durante la obtención de las muestras de saliva parotídea, se estimuló su salida mediante ácido cítrico al $2 \%$ colocado en el dorso de la lengua en intervalos de 30 segundos por 10 minutos. La saliva fue recolectada mediante el dispositivo Carlson-Crittenden, el cual fue colocado en la desembocadura del conducto de Stenon a nivel del 
TRIANA-REYES, S. A.; MARTÍNEZ-SANDOVAL, G.; RODRÍGUEZ-FRANCO, N. I.; CHAPA-ARIZPE, M. G.; RODRÍGUEZ-PULIDO, J. I.; MARTÍNEZ-GONZÁLEZ, G. I. \& RIEGATORRES, J. Índice de placa bacteriana, índice gingival y prueba de silometría en pacientes con síndrome de sjögren primario y secundario. Int. J. Odontostomat.,15(2):449-453, 2021.

primer molar superior, donde se depositaba la saliva parotídea en un microtubo de $2 \mathrm{ml}$ colocado sobre un contenedor con hielo (Navazesh \& Kumar, 2008). La evaluación de la sialometría se realizó a los 3 y 5 min.

Consideraciones éticas. El estudio fue aprobado por el Comité de Bioética de la Facultad de Odontología de la Universidad Autónoma de Nuevo León. Una vez que el paciente aceptó participar en el estudio y antes de realizar la historia clínica, se solicitó firmar el consentimiento informado.

Análisis de datos. Se realizaron Pruebas t student, de diferencia de medias, con un $95 \%$ de confiabilidad (IBM SPSS Statistics, Versión 20, USA y Microsoft Excel 2010).

\section{RESULTADOS}

En el grupo de pacientes con SS secundario las enfermedades asociadas con mayor frecuencia fueron: artritis reumatoide, lupus eritematoso sistémico y esclerosis sistémica. Al momento de participar en el estudio, los pacientes con SS primario y secundario se encontraban bajo tratamiento a base de inmunosupresores, analgésicos antiinflamatorios esteroideos y ácido fólico.

La evaluación periodontal resultó con presencia de bolsas periodontales y pérdida de inserción clínica, tanto el grupo con SS primario como el grupo con SS secundario, por lo que fueron diagnosticados con periodontitis crónica.

Los pacientes con SS primario mostraron tener mayor índice de placa $(3,53 \pm 0,5954)$ e índice gingival $(2,41 \pm 0,2608)$ en comparación de los pacientes del grupo de SS secundario, en el cual se encontró una media menor en el índice de placa $(1,62 \pm 0,3795)$ y en el índice gingival $(1,48 \pm 0,3994)$, con diferencia significativa $(p=0,0001)($ Tabla I).

Los pacientes con SS primario mostraron menor flujo salival a los 3 y 5 minutos que los pacientes con Síndrome de Sjögren secundario (Tabla II).

\section{DISCUSIÓN}

La enfermedad periodontal es una condición inflamatoria crónica causada por la placa dentobacteriana (Fernández-Plata et al., 2017) caracterizada por una serie de hallazgos clínicos y alto índice gingival e índice de placa (Matarasso et al., 2015), donde se ha encontrado relación con enfermedades autoinmunes como la artritis reumatoide (Triana et al., 2016).

En relación a la afección sobre el periodonto en el SS. Antoniazzi et al. encontraron que existe un mayor número de piezas perdidas en pacientes con SS primario y secundario, en comparación con pacientes sanos, así como un aumento en el índice gingival (65 $\%$ ) e índice de placa (75\%), profundidad de bolsa, nivel de inserción clínico y sangrado al sondeo (Antoniazzi et al.), al igual que otros estudios donde se encontraron mayores niveles de índice gingival e índice de placa en SS, que en pacientes sanos (Najera et al., 1997; Le Gall et al., 2016). A diferencia de Boutsi et al. (2000) quienes encontraron que no existen diferencias significativas en la condición dental y periodontal de pacientes con SS comparados con pacientes sanos, sin embargo las condiciones orales relacionaron negativamente con la edad, donde a pesar que el flujo salival fue considerablemente menor en SS, tenían mejores hábitos de higiene oral que en sujetos sanos.

Tabla I. Evaluación del índice de placa e índice gingival en pacientes con síndrome de Sjögren primario y secundario.

\begin{tabular}{lccccc}
\hline & \multicolumn{2}{c}{ Sjögren primario } & \multicolumn{2}{c}{ Sjögren secundario } & Valor $\mathrm{p}$ \\
& Media & $\mathrm{DE}$ & $\mathrm{Media}$ & $\mathrm{DE}$ & \\
\hline Índice gingival & 2,41 & 0,2608 & 1,48 & 0,3994 & 0,0001 \\
Índice de placa & 3,53 & 0,5954 & 1,62 & 0,3795 & 0,0001 \\
\hline
\end{tabular}

Tabla II. Evaluación de la sialometría (3 y 5 min) en comparación entre grupos.

\begin{tabular}{cccccc} 
& \multicolumn{2}{c}{ Sjögren primario } & \multicolumn{2}{c}{ Sjögren secundario } & Valor $p$ \\
& Media & DE & Media & DE & \\
\hline 3 min & 17,8 & 15,55 & 30,7 & 4,14 & 0,0104 \\
5 min & 14,5 & 13,17 & 26,9 & 4,15 & 0,0054 \\
\hline Valor $\mathrm{p}$ & & 0,1184 & & & 0,0001 \\
\hline
\end{tabular}


TRIANA-REYES, S. A.; MARTÍNEZ-SANDOVAL, G.; RODRÍGUEZ-FRANCO, N. I.; CHAPA-ARIZPE, M. G.; RODRÍGUEZ-PULIDO, J. I.; MARTÍNEZ-GONZÁLEZ, G. I. \& RIEGATORRES, J. Índice de placa bacteriana, índice gingival y prueba de silometría en pacientes con síndrome de sjögren primario y secundario. Int. J. Odontostomat.,15(2):449-453, 2021.

Sin embargo también se ha encontrado que el índice de placa es menor en pacientes con SS primario, comparado con pacientes con xerostomía, sin diferencia entre el índice gingival, índice de sangrado al sondeo y profundidad de bolsa entre ambos grupos (Pers et al.); al igual que en un estudio sobre las condiciones periodontales de pacientes son SS primario, secundario y pacientes sanos, donde además se comprobó que no hay diferencia entre la presencia de microorganismos periodontopatógenos (Kuru et al., 2002). En contraste con el presente estudio, los pacientes con SS primario presentaron mayor índice de placa e índice gingival comparados con pacientes con SS secundario, ambos grupos con periodontitis.

Los pacientes con síndromes de Sjögren primario mostraron menor cantidad de saliva a los 3 y 5 minutos, en comparación con los pacientes con síndrome de Sjögren secundario. La cantidad de placa bacteriana mayor en pacientes con SS primario y un flujo salival disminuido, hacen referencia a las funciones de la saliva como primera línea de defensa contra el ataque bacteriano y viral a la cavidad bucal (Marsh et al., 2016), además de lubricar, limpiar, facilitar el habla; ayudar al gusto, la masticación, la deglución y comenzar la digestión de los alimentos (Proctor, 2016).

Un estudio realizado por Tseng et al. (1990), encontraron un índice de placa de $0,50 \pm 0,35$ y un índice gingival de $0,98 \pm 0,36$, sin embargo no hace distinción entre SS primario y secundario. En el presente estudio, el índice de placa e índice gingival mostraron diferencias significativas y mayores en pacientes con SS primario, con altas cantidades de placa bacteriana e inflamación severa, comparados con pacientes con SS secundario, presentando bajas cantidades de placa bacteriana e inflamación leve. Una revisión sistemática muestra que existe diferencia significativa en el índice gingival en pacientes con SS y pacientes sanos, sin diferencia significativa en el índice de placa y el índice de sangrado (De Goés Soares et al., 2018).

\section{CONCLUSIÓN}

Los pacientes con SS primario y SS secundario fueron diagnosticados con periodontitis crónica. El índice de placa e índice gingival fueron mayores en pacientes con SS primario que en pacientes con SS secundario. La prueba de sialometría fue mayor en pacientes con SS secundario.
AGRADECIMIENTOS. A CONACYT por la beca otorgada.

TRIANA-REYES, S. A.; MARTÍNEZ-SANDOVAL, G.; RODRÍGUEZ-FRANCO, N. I.; CHAPA-ARIZPE, M. G.; RODRÍGUEZ-PULIDO, J. I.; MARTÍNEZ-GONZÁLEZ, G. I. \& RIEGA-TORRES, J. Bacterial plate index, gingival index, and silometry testing in patients with primary and secondary sjögren's syndrome. Int. J. Odontostomat.,15(2):449-453, 2021.

ABSTRACT: Sjögren's syndrome (SS) is a chronic autoimmune disease present in $0.1-3.0 \%$ of the population, in which the salivary glands are involved, bringing with it oral manifestations such as dental caries and periodontal disease. The aim of the present study was to evaluate the plaque index, gingival index and the sialometry test in patients with primary and secondary Sjögren's syndrome. Forty patients with primary $S S(n=20)$ and secondary SS $(n=20)$, both groups diagnosed with chronic periodontitis, were evaluated clinically. The Quigley-Hein plaque index modified by Turesky, the gingival index of Löe and stimulated sialometry test collected from parotid gland by using Carlson-Crittenden device were recorded. The patients with primary SS had plaque index $(3.53 \pm 0.5954)$ and gingival index $(2.41 \pm$ $0.2608)$. The patients with secondary SS had a plaque index $(1.62 \pm 0.3795)$ and gingival index (1.48 \pm 0.3994$)$, with significant difference between both groups. The present study concludes that plaque index and gingival index were higher in patients with primary SS than patients with secondary SS. Sialometry test were higher in patients with secondary SS.

KEY WORDS: Sjögren's syndrome, plaque index, gingival index, sialometry test, periodontitis.

\section{REFERENCIAS BIBLIOGRÁFICAS}

Antoniazzi, R. P.; Miranda, L. A.; Zanatta, F. B.; Islabão, A. G.; Gustafsson, A.; Chiapinotto, G. A. \& Oppermann, R. V. Periodontal Conditions of Individuals With Sjögren's Syndrome. J. Periodontol., 80(3):429-35, 2009.

Berkey, D. \& Scannapieco, F. A. Medical considerations relating to the oral health of older adults. Spec. Care Dent., 33(4):164-76, 2013.

Boutsi, E.A.; Paikos, S.; Dafni, U. G.; Moutsopoulos, H. M. \& Skopouli, F. N. Dental and periodontal status of Sjögren's syndrome. J. Clin. Periodontol., 27(4):231-5, 2000.

De Goés Soares, L.; Rocha, R. L.; Bagordakis, E.; Galvão, E. L.; Douglas-de-Oliveira, D. W. \& Falci, S. G. M. Relationship between sjögren syndrome and periodontal status: A systematic review. Oral Surg. Oral Med. Oral Pathol. Oral Radiol. Endod., 2018;125(3):223-31, 2018

Deepak, D. \& Gopakumar, R. Xerostomia, its association with oral manifestation and ocular involvement: a clinical and biochemical study. J. Indian Acad. Oral Med. Radiol., 23(4):513-7, 2011.

Donat, F. J. S.; Jordá, L. M. \& Mihi, V. M. Tratamiento de la boca seca: puesta al día. Med. Oral, (4):273-9, 2004. 
Fernández-Plata, R.; Olmedo-Torres, D.; Martínez-Briseño, D.; González-Cruz, H.; Casa-Medina, G. \& García-Sancho, C. Días de estancia hospitalaria (DEH) en pacientes con enfermedades respiratorias (ER) y enfermedad periodontal (EP). Gac. Med. Mex., 153:31-5, 2017.

Goldblatt, F. \& O'Neill, S. G. Clinical aspects of autoimmune rheumatic diseases. Lancet, 382(9894):797-808, 2013.

González, S.; Sung, H.; Sepúlveda, D.; González, M. \& Molina, C. Oral manifestations and their treatment in Sjögren's syndrome. Oral Dis., 20(2):153-61, 2014.

Jensen, S. B.; Pedersen, A. M. L.; Vissink, A.; Andersen, E.; Brown, C.G.; Davies, A. N.; Dutilh, J.; Fulton, J. S.; Jankovic, L.; Lopes, N. N.; et al. A systematic review of salivary gland hypofunction and xerostomia induced by cancer therapies: management strategies and economic impact. Support Care Cancer, 18(8):1061-79, 2010.

Kuru, B.; McCullough, M. J.; Yilmaz, S. \& Porter, S. R. Clinical and microbiological studies of periodontal disease in Sjögren's syndrome patients. J. Clin. Periodontol., 29(2):92-102, 2002.

Le Gall, M.; Cornec, D.; Pers, J. O.; Saraux, A.; Jousse-Joulin, S.; Cochener, B.; Roguedas-Contios, A. M.; Devauchelle-Pensec, V. \& Boisramé, S. A prospective evaluation of dental and periodontal status in patients with suspected Sjögren's syndrome. Joint Bone Spine, 83(2):235-6, 2016.

Löe, $\mathrm{H}$. The gingival index, the plaque index and the retention index systems. J. Periodontol., 38(6):610-6, 1967.

Marsh, P. D.; Do, T.; Beighton, D. \& Devine, D. A. Influence of saliva on the oral microbiota. Periodontol., 2000, 70(1):80-92, 2016.

Matarasso, M.; Iorio-Siciliano, V.; Blasi, A.; Ramaglia, L.; Salvi, G. E. \& Sculean, A. Enamel matrix derivative and bone grafts for periodontal regeneration of intrabony defects. A systematic review and meta-analysis. Clin. Oral Investig., 19(7):1581-93, 2015.

Najera, M. P.; Al-Hashimi, Plemons, J. M.; Rivera-Hidalgo, Rees T. D.; Haghighat N. \& Wright J. M. Prevalence of periodontal disease in patients with Sjögren's syndrome. Oral Surg. Oral Med. Oral Pathol. Oral Radiol. Endod., 83(4):453-7, 1997.

Navazesh, M. \& Kumar S. K. S. Measuring salivary flow: Challenges and opportunities. J. Am. Dent. Assoc., 139:35S-40S, 2008.

Pers, J.; d'Arbonneau, F.; Devauchelle, V.; Saraux, A.; Pennec, Y. \& Youinou, $P$. Is periodontal disease mediated by salivary baff in Sjögren's syndrome? Arthritis Rheum., 52(8):2411-4, 2005.

Proctor, G. B. The physiology of salivary secretion. Periodontol. 2000, 70(1):11-25, 2016.

Rodríguez-Pulido, J.; Martínez-Sandoval, G.; Rodríguez-Franco, N.; Chapa-Arizpe, M.; Riega-Torres, J. \& Garza-Elizondo, M. Salivary stimulation by prolonged release of pilocarpine in Sjögren's syndrome. J. Oral Res., 6(3):65-9, 2017.

Rodríguez, J. I.; Martínez, G.; Rodríguez, N. I.; Chapa, M. G. \& Solís, J. M. Terapia farmacológica y avances terapéuticos en xerostomía e hiposalivación. Rev. ADM, 74(5):221-3, 2017.

Rodríguez, J.; Martínez, G.; Rodríguez, N.; Chapa, M. \& Solis, J. Dental perspective on Sjögren's syndrome: literature review. J. Oral Res., 4(3):211-22, 2015a.

Rodríguez, J.; Sánchez, R.; Garza, M.; Nakagoshi, M.; Solis, J.; Arévalo, K. \& Garza, E. Salivary stimulation by prolonged release of pilocarpine using films in diabetic rats. J. Oral Res., 4(2):1038, 2015b.

Shahane, A. \& Patel, R. The epidemiology of Sjögren's syndrome. Clin. Epidemiol., 6(1):247-55, 2014.

Triana, S. A.; Rodríguez, J. I.; Garza, B. R.; Martínez, G. \& Rodríguez, N. I. Relación entre periodontitis y artritis reumatoide. Revisión de literatura. Odontol. Act., 13(160):44-7, 2016.

Tseng, C. C. Wolff; L. F. Rhodus, N. \& Aeppli, D. M. The periodontal status of patients with Sjögren's syndrome. J. Clin. Periodontol., 17(5):329-30, 1990.
Turesky, S.; Gilmore, N. D. \& Glickman, I. Reducen plaque formation by the chloromethyl analogue of vitamine C. J. Periodontol., 41(1):41-3, 1970.

Yoshimoto, K.; Fujimoto, T.; Itaya, A.; Miyaoka, T.; Sakuramoto, S.; Yamauchi, A.; Takeda, M.; Kasai, T.; Nakagawara, K.; Nonomura, A.; et al. Involvement of autoimmunity to REG, a regeneration factor, in patients with primary Sjögren's syndrome. Clin. Exp. Immunol., 174(1):1-9, 2013.

Dirección para correspondencia:

Silvia Arely Triana-Reyes

Posgrado de Periodoncia

Facultad de Odontología

Universidad Autónoma de Nuevo León

E. Aguirre Pequeño y Silao

Col. Mitras Centro C.P. 64460

Monterrey, Nuevo León

MÉXICO

E-mail: silvia_triana@hotmail.com 